\title{
6-Hydroxydopamine lesions in the medial prefrontal cortex of rats exposed to a peak-interval procedure
}

\author{
Mirari Elcoro ${ }^{1}$, Tina Thompson ${ }^{2}$, Scot P. Kelly ${ }^{1}$, Rhiannon Pegan ${ }^{1}$, and Carlos F. Aparicio ${ }^{3}$ \\ 1. Armstrong Atlantic State University, Savannah, GA, United States \\ 2. Mercer School of Medicine, Savannah, GA, United States \\ 3. Salem State University, Salem, MA, United States
}

\begin{abstract}
Impaired temporal control is symptomatic of several neurological disorders; recently, it has been implicated in schizophrenia. An animal model of schizophrenia using 6-hydroxydopamine(6-OHDA) infused to the medial pre-frontal cortex (mPFC) was employed to examine its effects on temporal control. Twelve rats were trained on a peak-interval procedure(PIP) until stable patterns ofbehavior were obtained. Rats infused with 6-OHDAresponded less during peak trials and their peak functions were flatter than sham rats. These results are consistent with similar studies with transgenic mice with increased striatal dopamine D2 receptor activity. Lesions in the $\mathrm{mPFC}$ decreased motivation to respond in a PIP. These effects may be considered analogous to negative symptoms of schizophrenia. Keywords: dopamine, peak-interval procedure, rats, temporal control.
\end{abstract}

Received 12 November 2013; received in revised form 16 January 2014; accepted 17 January 2014. Available online 20 May 2014.

\section{Introduction}

Accurate use of time is involved in many of our daily activities. The study of how behavior is modified by changes in time is referred to as temporal control or timing. Temporal control deals with intervals ranging from seconds to minutes and is defined as the sensitivity of behavior associated with temporal organization of environmental events (Catania, 1991). In the operant conditioning laboratory, several schedules of reinforcement may be arranged to examine how behavior is organized according to time. For example, in a fixed-interval (FI) schedule of reinforcement, a reinforcer is delivered when an operant response occurs after a fixed time has elapsed (Ferster \& Skinner, 1957). A variation of the FI schedule, used in the present study, is the peak-interval procedure (PIP, Roberts, 1981). The PIP involves opportunities to earn reinforcers following an FI schedule, intermixed with longer trials in which

Mirari Elcoro, and Rhiannon Pegan, Armstrong Atlantic State University - Psychology. Scot P. Kelly, Armstrong Atlantic State University - Biology. Tina Thompson, Mercer School of Medicine. Carlos F. Aparicio, Salem State University - Psychology. Correspondence regarding this article should be directed to: Mirari Elcoro, Ph.D., Assistant Professor, Department of Psychology, Armstrong Atlantic State University, 11935 Abercorn St. Savannah, GA 31419-1997. Tel.: 912 3442927. Fax.: 912-3443484. E-mail: mirari.elcoro@armstrong.edu no reinforcers are delivered (i.e., peak trials). Both types of trials (i.e., FI and peak trials) are randomly arranged and each is separated by an inter-trial-interval (ITI). The resulting patterns of behavior after repeated exposure to the peak trials consist of a progressive increase of responding that peaks around the target time (i.e., the FI value) and then decelerates.

The PIP yields comparable behavioral patterns across several species (Lejeune,1990) making it an adequate procedure to develop an animal model to better understand aspects of human behavior. More specifically, the PIP has been extensively used to study the role of dopamine (DA) in temporal control and has implications in understanding various degenerative disorders such as Parkinson's Disease (see Balci et al. $2010 \mathrm{~b}$ for a comprehensive review). More recently, the PIP has been proposed to study behavioral alterations and neurological underpinnings of schizophrenia (Balci et al., 2010b). Some of these studies (Balci et al., 2010a, Simpson et al., 2011; Ward et al., 2009, 2011) used transgenic mice to model DA alterations that underlie symptoms of schizophrenia. In one of these studies Balci et al. (2010) used mice that underexpress the DA transporter (DAT), which in turn have higher levels of extracellular DA. In a PIP with two intervals (FI 30 and $45 \mathrm{~s}, 120$-s peak trials) the transgenic mice responded earlier during the peak trials relative to controls. The authors concluded that elevated extracellular DA increased the levels of motivation for responding in the procedure. 
Ward et al. (2009) used transgenic mice that selectively overexpress the striatal D2 receptor and a PIP with a single interval (FI $12 \mathrm{~s}$, peak trials of $48 \mathrm{~s}$ ) and varying probabilities of reinforcement. In the PIP, the transgenic mice showed a significant reduction in overall responding relative to controls. In addition, as the probability for reinforcement increased, the timing functions obtained with responding during the peak trials recovered. Ward et al. suggested the finding was related to a motivational deficit, analogous to negative symptoms (i.e., avolition) of schizophrenia (Ward et al. 2011). Similarly, other procedures (e.g., finger tapping task, Carroll et al., 2009) have also shown that patients diagnosed with schizophrenia exhibit distortions in temporal control (Elvevåg, Weinberg, \& Goldberg, 2002; Tysk, 1983).

Animal models have also been used to examine cognitive symptoms of schizophrenia such as defects in working memory (Ward et al., 2011) which depend on the functioning of the prefrontal cortex (PFC, Heidbreder \& Groenewegen, 2003; Olton, 1989). Planning and execution of complex behaviors are functions of the PFC; specifically the dorsal part of the medial PFC (mPFC) is involved in the temporal patterning of behavioral sequences (Heidbreder \& Groeneweger; Valencia-Torres et al., 2011, 2012). The alterations of temporal control described above may be considered part of the cognitive symptoms in schizophrenia (Balci et al., 2010).

Brain lesions may be performed following different methods and a few have been used to examine ongoing behavior maintained in a PIP. For example, Olton (1989), Olton et al. (1988), and Meck (2006) used aspiration to remove most of the frontal cortex (FC) and examined the effects of such lesions on performance in a PIP. In these three studies an attempt was made to leave the cingulate cortex and underlying fiber tracts of the corpus callosum intact (see studies for more details). Olton (1989) observed selective alterations of temporal control: the functions formed during the peak trials were shifted to the right, which indicates responding happened slightly later than prior to the FC lesions when two intervals (FI $10 \& 20 \mathrm{~s}$, peak trials of $130 \mathrm{~s}$ ) were used in a PIP. Responding shifted to the right more pronouncedly in the FI $20 \mathrm{~s}$ trials and during peak trials. In this same study, the number of days to recover from the lesions was not specified. Olton et al. (1988) employed a PIP as the one previously described. After the FC lesions, 19 sessions of postoperative training were conducted and peak times increased by $22 \%$ relative to preoperative levels. Meck (2006) found that dopaminergic drugs (haloperidol, methamphetamine and CCK-8S) did not alter temporal control in rats with frontal cortex lesions. Postoperative training was conducted 3 months after the lesions and no assessment without drugs post lesions was presented.

Another type of lesion, preferred over aspiration for its specificity in targeting DA neurons, is stereotaxic guided infusions of 6-hydroxydopamine (6-OHDA), a neurotoxin that selectively destroys DA neurons. This lesion model in which 6-OHDA is infused in the MPFC, has contributed to the generation of a modified DA hypothesis for schizophrenia. More specifically in this 6-OHDA model, the depletion of dopaminergic neurons reaches the ventral tegmental area destroying the DA mesocortical system and in turn increases levels of DA in the striatum and nucleus accumbens (NAC) (Li et al., 2010).

Li et al. (2010) performed bilateral infusions of 6-OHDA in the mPFC of adolescents rats and adult rats and found a significant decrease in grooming and social behavior. The researchers paralleled these findings to negative symptoms observed in schizophrenia. It is important to note that the negative symptoms of schizophrenia have received less attention than positive symptoms within research that employs animal models to better understand this disorder (Jones, Watson, \& Fone, 2011).

Some operant conditioning procedures, other than the PIP, in conjunction with 6-OHDA lesions of the mPFC have been conducted by Martin-Iverson, Szostak, and Fibiger (1986) and Sokolowski and Salamone (1994). The former authors found that mPFC lesions using 6-OHDA failed to influence intravenous selfadministration of cocaine. Sokolowski and Salamone found that rats infused with 6-OHDA responded more and earned less reinforcers than sham rats when exposed to a differential-reinforcement-of-low rates schedule of reinforcement in which behavioral inhibition is required to maximize the number of reinforcers earned. These two studies examined behavior of rats 3 days after surgery, and conducted the corresponding procedures for 10-14 days to assess the effects of such lesions on their corresponding measures.

To our knowledge, no study has yet evaluated the effects of 6-OHDA lesions as a model of schizophrenia using a PIP. If distortion of temporal control is implicated in schizophrenia, then it is worth examining the 6-OHDA model using a PIP. The present experiment examined the effects of 6-OHDA lesions in the mPFC of rats trained in a PIP (FI 30 s, 90-s peak trials, and 10-s ITIs). The time of recovery from surgery was longer (5 weeks) than most of the previous studies described. A longer recovery time was used to ensure that the lesions had taken effect (Kostrzewa \& Jacobowitz, 1974) and that full recovery from surgery and anesthesia would not account for the effects observed. Additionally, training after the lesions was extended ( 38 sessions) to examine if recovery of behavior (to levels observed prior to the lesions) occurred after the lesions.

\section{Method}

\section{Subjects}

Twelve male Sprague Dawley (Charles River, $\mathrm{NC}$ ) rats approximately 8 months old at the start of the 
experiment, with previous experimental history with schedules of positive reinforcement, served as subjects. Rats were maintained at $85 \%$ of their free-feeding body weight, each housed individually with water available in their home cages. Illumination was kept in a 12-hr light /dark cycle and temperature and humidity were kept constant. All experimental procedures were approved by the Animal Care and Use Committees at Armstrong Atlantic State University and Mercer University School of Medicine.

\section{Apparatus}

Four modular standard operant conditioning chambers for rats each kept in an isolation cubicle (Coulbourn Instruments, Whitehall, PA) were used. Operant chambers were $31 \mathrm{~cm}$ by $26.4 \mathrm{~cm}$ by $32.8 \mathrm{~cm}$. Each chamber had modular walls with filler panels and one standard response lever each which required a force of $0.25 \mathrm{~N}$ to depress. The standard stainless steel response lever protruded $2 \mathrm{~cm}$ from the wall, was 3.5 $\mathrm{cm}$ wide, was at a height of $6.6 \mathrm{~cm}$ from the bar floor and was $3.2 \mathrm{~cm}$ from the feeder trough on the same wall. A houselight was positioned in the upper-right corner of the same wall as the lever and the feeding trough. There were two halves of the trough, one for liquid reinforcers on the left and one for dry reinforcers on the right. Only the dry reinforcer half of the trough was used for this experiment and was illuminated each time a reinforcer was delivered. Sucrose-based pellets (45$\mathrm{mg}$ ) functioned as reinforcers. Experimental procedures and real-time data collection were programmed using Graphic State (Coulbourn Instruments) software.

\section{Procedure}

Fixed-interval (FI) schedule pretraining. During these sessions, a FI 30-s schedule was in effect. The schedule proceeded as follows: the first press after 30 $\mathrm{s}$ resulted in the delivery of a pellet, the houselight illuminating the chamber turned off during the delivery of every reinforcer, simultaneously the magazine light was on for $3 \mathrm{~s}$, and then a new FI commenced. Sessions ended after 40 reinforcers were delivered. Each rat was exposed to 22 sessions.

Preoperative peak-interval procedure (PIP) training. Rats were trained at approximately the same time, 5 days a week, in a peak-interval procedure (PIP). The procedure consisted of 42 FI 30-s trials and eighteen 90-s peak trials. In the FI 30-s trials, reinforcers were available at the first press after $30 \mathrm{~s}$ had elapsed. The houselight was on except for when the reinforcer was delivered (the magazine light was illuminated at this point). In the peak trials the houselight remained on for $90 \mathrm{~s}$ and no reinforcers were delivered. All trials were separated by a 20 -s intertrial interval (ITI) in which the houselight and magazine light were turned off and lever presses had no programmed consequences. Sessions lasted approximately $70 \mathrm{~min}$ and each rat was exposed to 38 sessions prior to surgery.
Postoperative PIP training. This phase of the experiment began 5 weeks after surgery. Training was identical to that described above for preoperative training and the same numbers of sessions were conducted.

DA depletion by injection of 6-OHDA. The twelve rats were divided into two groups: six rats were infused with 6-OHDA (6-OHDA group) and five rats were infused with vehicle (sham group). Groups were formed counterbalancing response rates. One rat was excluded from the study, prior to surgery, due to illness. The rats were anesthetized with a ketamine/xylazine (800 mg/120 mg; $1 \mathrm{ml} / \mathrm{kg}$ ) mixture, shaved, and placed in a stereotaxic apparatus (Stoelting,Wood Dale, IL) on a thermal pad to maintain optimal body temperature. Xylocaine was added to the shaved skin prior to the initial incision. Burr holes were made in the skull and the dura mater removed. Solutions of either 6-OHDA or ascorbate vehicle were injected through a Hamilton syringe into the medial prefrontal cortex (mPFC) at four locations (AP $+2.6 \mathrm{~mm}$, $+2.3 \mathrm{~mm}$ from bregma, $\mathrm{L} \pm$ $0.75 \mathrm{~mm}, \mathrm{DV}-3.4 \mathrm{~mm},-3.6 \mathrm{~mm}$ from skull). Each rat received four infusions, two per side of either 6-OHDA or vehicle. Each injection of 6-OHDA consisted of $2.0 \mathrm{mg}$ 6-OHDA/500 $\mu \mathrm{l}$ 0.02\% ascorbate-vehicle (10 $\mathrm{mg} / 50 \mathrm{ml}$ ) prepared in an eppendorf tube and covered in foil. Infusions of $1 \mu \mathrm{l}$ of 6 -OHDA or vehicle $(0.02 \%$ ascorbate saline) were conducted at a rate of $0.2 \mu \mathrm{l} / \mathrm{min}$. After each infusion was completed, the Hamilton syringe was left in place for three min before slow removal. This was repeated with the other three sites. Bone wax was used to seal the skull and skin was closed with surgical wound clips. Each rat was placed in a clean, separate cage on a thermal pad after surgery.

Immunocytohistochemistry. Lesions were further examined using immunocytochemistry. To do this, after the experiment was completed rats were sacrificed, perfused, and brains were extracted and preserved with a fixative. A vibratome was used to slice the areas corresponding to the $\mathrm{mPFC}$ and the striatum. The thickness of all slices was 80 microns. The visibility of the tyrosine hydroxilase neurons was made possible via immunohistochemical staining (Vectastain ABC Kit, Vector Labs, Burlingame, CA). Once stained, slices were mounted on glass using a mounting medium. Images of tyrosine hydroxylase fibers were captured using an inverted microscope (Olympus 1x71/1x51) with image capture software. Images of the $\mathrm{mPFC}$ were magnified and printed. Four observers were trained to identify tyrosine hydroxylase fibers and were given 33 unidentified images (three per rat). A transparent grid with 135 (13 $\mathrm{mm}$ by $13 \mathrm{~mm}$ ) squares was superimposed on each printed image to guide counting. The observers counted the total presence of tyrosine hydroxylase fibers per square on each image. Observers used a mechanical tally counter to keep track of all counts per image. The outcomes of the counts remained anonymous and were compiled and compared. Each observer produced a total count per image. 


\section{Results}

Before the lesions were performed, all rats $(n=11)$ were responding similarly, with mean peak times of 30.41 $\mathrm{s}(S E M=0.29)$ and mean overall response rates (for peak trials $)$ of $27.96 \mathrm{resp} / \mathrm{min}(S E M=0.33)$. The remaining data analysis was focused on performance during peak trials.

Comparisons between 6-OHDA and sham groups were made calculating response distributions during peak trials in 5-s bins, as shown in Figure 1. Relative response rates were obtained by dividing the overall response rates for each 5-s bins (total of 18 bins for a 90-s peak trial, $\mathrm{x}$ axis) by the maximum response rate during baseline. Response rates were obtained for each rat and based on the last five sessions of each condition. Figure 1 shows the shape of the peak functions for each

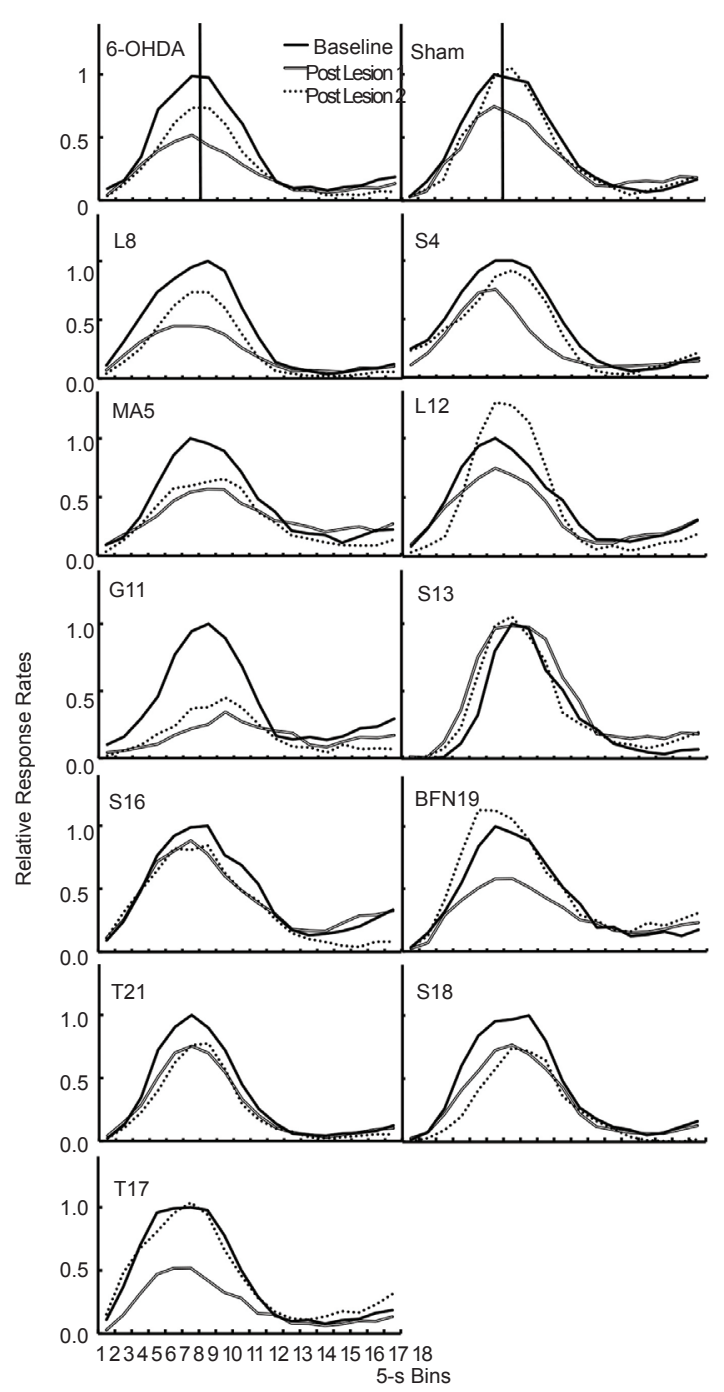

Figure 1. Relative response rates for each group (medians for 6-OHDA and sham; top two graphs) and for individual rats (below each corresponding top graph). In each graph, solid lines represent performance for the last five sessions prior to surgery (baseline), empty lines represent performance for the first five sessions after surgery (PL 1, post lesion 1), and dashed lines represent performance for the last five sessions after surgery (PL 2, post lesion 2). rat and also from the group (medians, two top graphs of Figure 1). Post lesion 1 (PL 1) corresponds to the first five sessions of training in the PIP after the lesions and post lesion 2 (PL 2) corresponds to the last five sessions of the total 38 sessions of post-lesion training.

A mixed ANOVA (GLM, repeated measures, SPSS 19) using time (Baseline, PL1, PL2) as within-subjects variable and group (6-OHDA, sham) as betweensubjects variable was carried out with the means of the following dependent variables: relative response rates (shown in Figure 1), peak rates, peak times, area under the curve (AUC), and post-reinforcement pauses (PRP). Additionally, parameters related to peak rates and peak times were obtained using a fit to a Gaussian function (amplitude version of Gaussian peak function, Origin ${ }^{\circledR}$ 8.5) and were also analyzed in this way.

The means for relative response rates were calculated by averaging the last five sessions across bins for each rat. The mixed ANOVA for relative response rates yielded no significant differences between groups $(F=1.65)$, but a significant time effect $(F=7.29$, d.f. $=2, p<.05)$. The interaction of group and time was not significant. Pairwise comparisons indicated that the difference between mean relative rates in baseline and PL1 were significant $(p<.05)$ and the difference between PL 1 and PL 2, and Baseline and PL 2 were not significant. Further examination of these data indicate that after 38 sessions, recovery of responding occured in sham rats and not in 6-OHDA rats. More specifically, relative response rates for sham rats resembled those prior to the lesions and in some cases (Rats L12, S13, and BFN19) exceeded these baseline levels. Within group analysis for the 6-OHDA group, using paired samples tests (two tailed) yielded significant differences between baseline and PL1 $(t=4.36$, d.f. $=5, p<.05)$ and between baseline and PL $2(t=3.58$, d.f. $=5, p<.05)$. Following the same analysis for the sham group, there were no significant differences across the same conditions (baseline \& PL 1, $t$ $=1.47$; baseline \& PL 2, $t=.68$ ).

Mean peak rates (i.e., maximum number of responses in 1-s bins averaged across trials per session) were obtained by averaging these measures across the last five sessions of each time condition per rat. In the 6-OHDA group, mean peak rates decreased more than in shams, relative to baseline levels. For rats in the 6-OHDA group, peak rates during PL 1 decreased 33\% relative to baseline, and during PL2, still they were 19\% lower than baseline. In contrast, mean peak rates of sham rats decreased 24\% during PL 1 and recovered almost completely to baseline, decreasing only $3 \%$. These results are consistent with those of relative response rates described above. The mixed ANOVA analysis using the same between and within variables previously indicated, yielded a significant time effect $(F=9.18$, d.f. $=2, p$ $<.05)$ and no significant effects for group $(F=.004)$. The interaction of group and time was not significant. Responding of sham rats, given extended training in the PIP, recovered to similar responding levels prior to mPFC lesions. Infusions of 6-OHDA prevented parallel 
levels of recovery, despite extended training in the PIP. Within group analysis with 6-OHDA yielded a significant difference between mean peak rates in baseline and PL1 $(t=3.22$, d.f. $=5, p<.05)$. The same analysis within the sham group yielded only one significant difference that was also between baseline and PL1 $(t=3.45$, d.f. $=4, p<.05)$. The same mixed ANOVA analysis was conducted with the parameter related to peak rate obtained by fitting a Gaussian curve to the data shown in Figure 1, and yielded no significant effects for group $(F$ $=.54)$ or time $(F=.78)$. When examining within group mean peak rates (parameter), in the sham group, there was a significant difference between peak rates between baseline and PL $2(t=3.73$, d. f. $=4, p<.05)$.

Mean peak times (i.e., bin with highest number of responses averaged across trials per session) remained unchanged when comparing baseline, PL1, and PL2. More specifically, for the 6-OHDA group, the mean peak times during baseline were $29.49 \mathrm{~s}(S E M=3.52)$, $29.51 \mathrm{~s}(S E M=3.59)$ during PL 1 , and $31.46(S E M=$ 3.56) during PL 2. For shams, the corresponding mean peak times were $31.52,31.66$, and 31.93 (SEM $=1.19$, $1.44,1.63)$. The mixed ANOVA for peak times yielded no significant effects for group $(F=.90)$ or time $(F=$ $.66)$. The same analysis using the related parameter to peak times (xc) obtained by a Gaussian fit paralleled the outcome previously described: no significant differences between group $(F=.66)$ or time $(F=.39)$.

Additional data analyses included calculating the AUC (following the theory-free method by Myerson, Green, \& Warusawitharana, 2001) for the peak functions shown on Figure 1. This measure was expressed as a proportion of the maximum AUC (values ranged from $0-1$ ) where decreases of the values indicate changes in the shape of the function. One difference between using this measure to examine peak functions in contrast to the measures provided above (i.e., peak rate, peak time) is that it includes the whole instead of a portion of the function. Figure 2 shows the percent change of the mean AUCs for each group. This measure reflects that recovery to baseline levels (represented by the horizontal dashed line) occur only in sham but not in 6-OHDA rats, as the values of the AUCs returned to baseline levels after training. This recovery was evident during post lesion 2 (PL 2) for the sham and not for the 6-OHDA rats. The mixed ANOVA using mean AUC yielded a significant time effect $(F=8.01$, d.f. $=2, \mathrm{p}$ $<.05$ ). To further examine this difference in recovery between each group, independent samples $t$ test were conducted; at PL 2 sham and lesion groups differed significantly (two-tailed, $t=2.60$, d.f. $=8, p<.05$ ).

Analysis of mean PRPs (as in Saulsgiver et al., 2006) yielded no significant differences between groups $(F=.37)$ or across time $(F=.93)$. As mentioned above, Gaussian functions were obtained (amplitude version of Gaussian peak function, Origin ${ }^{\circledR} 8.5$ ) using the medians of relative response rates shown in Figure 1. The results of these fits are shown in Figure 3. The parameters

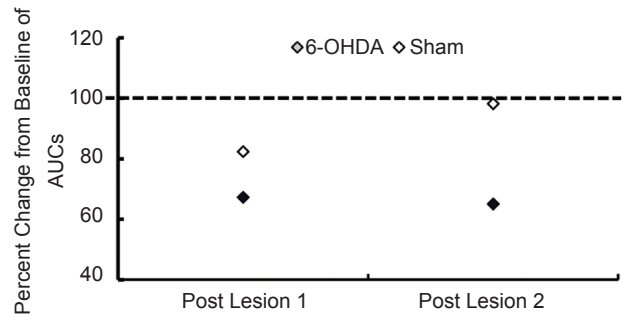

Figure 2. Percent change from baseline of AUCs for 6-OHDA rats (filled diamonds) and sham rats (open diamonds) during post lesion 1 and post lesion 2. Horizontal dashed line represents baseline.

for the median relative rates (y0, intercept, $\mathrm{xc}$, peak time, w, peak rate, A, amplitude, FWHM, area, and $\mathrm{R}^{2}$ goodness of fit) and for individual rats are presented in Tables 1 and 2 respectively. In general, the data obtained during peak trials was well described by the Gaussian fits given the obtained high values of best fit index $\mathrm{R}^{2}$ (see Tables $1 \& 2$ ).

Counts of tyrosine hydroxylase fibers (described in the Method section) were summed across the three images for the same rat for each observer resulting in an overall count for each rat per observer. High and significant Pearson correlation coefficients were obtained when the overall counts from the four observers were compared (observer $1 \& 2 r=.504, p$ $=.003$, observer $1 \& 3 r=.950, p<.001$; observer 1 $\& 4 r=.968, p<.001$; observer $2 \& 3 r=.525, p=$ .002 ; observer $2 \& 4 r=.551, p=.001$; observer $3 \& 4$ $r=.967, p<.001$ ) which reflect concordance between observations. Counts across observers were summed for each rat, and then average counts between 6-OHDA ( $M$ $=1400.00, M d=857.00, S E M=464.36, \operatorname{Min}=473.00$, $M a x=3427.00)$ and shams $(M=2500.80, M d=1542.00$, $S E M=674.24, \operatorname{Min}=1178.00, \operatorname{Max}=4282.00)$ were compared. In general, the mean (and median) number of counts was higher in sham rats than in those infused with 6-OHDA, which is indicative of decreased tissue concentration of DA in the mPFC areas infused with 6-OHDA. Although such difference in mean counts between groups was not statistically significant, it was in the expected direction. Images of the striatum used as controls, to ensure that the lesions had remained in the mPFC, were also examined and confirmed that tyrosine hydroxylase neurons were not affected in the striatum of any of the groups.

\section{Discussion}

Given enough training, responding of sham rats recovered to levels near baseline after vehicle infusions. Rats infused with 6-OHDA continued to respond generally at lower rates relative to their own baseline as shown in Figure 1, and did not show the same level of recovery as the rats in the sham group. One measure that reflects a general change in the shape of the whole function, the AUC, was significantly lower in the 6-OHDA than in the sham group during PL2, indicating 

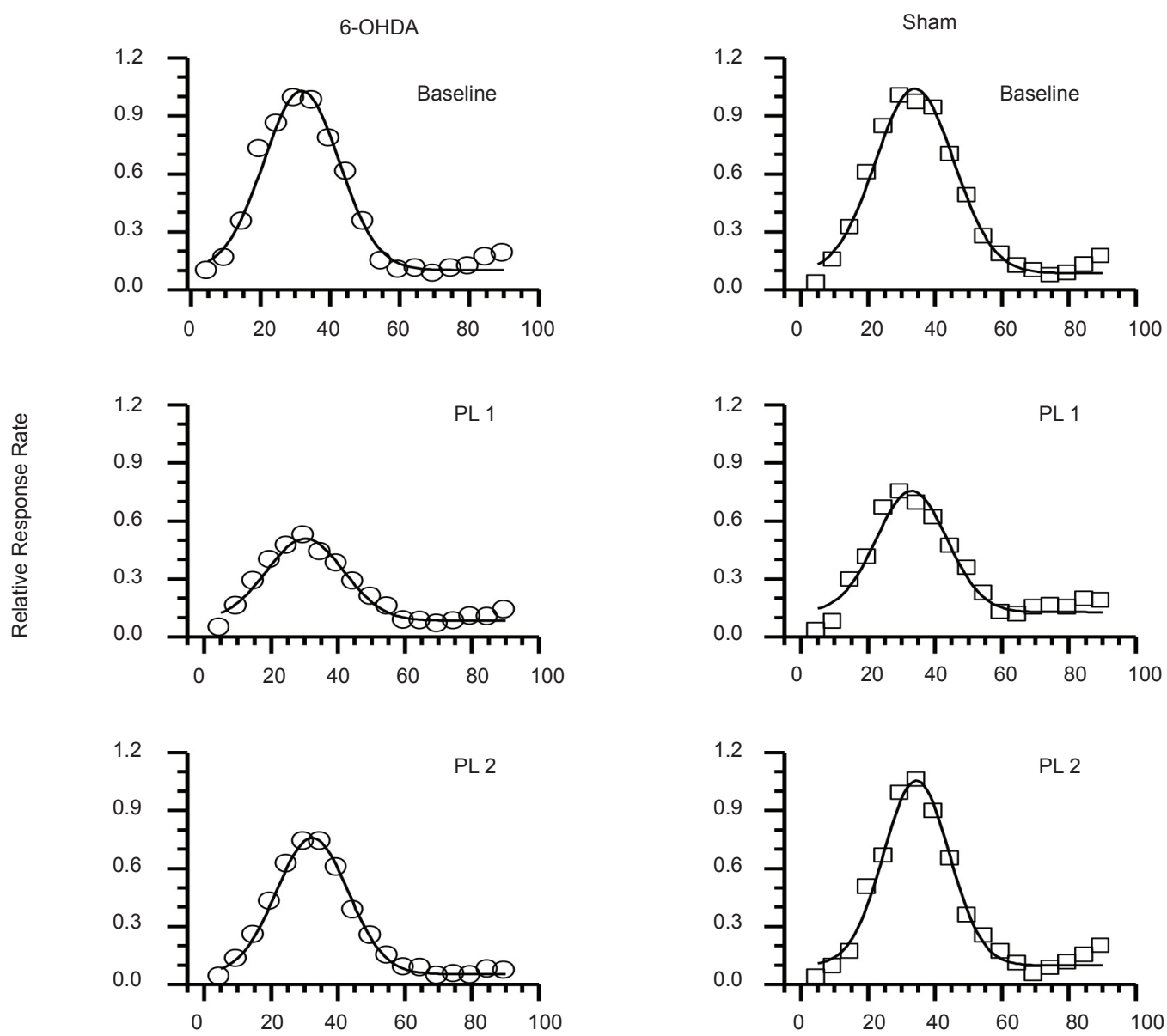

Bins (s)

Figure 3. Gaussian functions derived from relative response rates across 5-s bins for each experimental condition (Baseline, PL1 and PL 2) and for each group (6-OHDA and sham).

Table 1. Parameters of gaussian peak functions (correspond to Figure 3) derived from median relative response rates with corresponding standard errors (SE).

\begin{tabular}{llllllllllllll}
\hline Condition & Group & $\mathbf{y 0}$ & SE & $\mathbf{x c}$ & SE & $\mathbf{w}$ & SE & A & SE & FWHM & Area & $\mathbf{R}^{2}$ \\
\hline \multirow{2}{*}{ Baseline } & 6-OHDA & .102 & .021 & 31.787 & .459 & 10.863 & .564 & .927 & .037 & 25.581 & 25.232 & .974 \\
& Sham & .086 & .019 & 33.929 & .405 & 11.703 & .516 & .955 & .032 & 27.558 & 28.001 & .982 \\
& & & & & & & & & & & & & \\
PL1 & 6-OHDA & .084 & .013 & 30.165 & .618 & 11.546 & .766 & .423 & .022 & 27.189 & 12.249 & .958 \\
& Sham & .128 & .022 & 32.992 & .716 & 10.509 & .876 & .627 & .040 & 24.746 & 16.526 & .936 \\
PL2 & 6-OHDA & .053 & .007 & 32.290 & .202 & 10.741 & .248 & .704 & .013 & 25.293 & 18.964 & .995 \\
& Sham & .098 & .019 & 34.446 & .415 & 9.780 & .498 & .957 & .038 & 23.030 & 23.455 & .976 \\
\hline
\end{tabular}


Table 2. Parameters of gaussian peak functions (correspond to Figure 3) for individual rats derived from relative response rates with corresponding standard errors (SE).

\begin{tabular}{|c|c|c|c|c|c|c|c|c|c|c|c|c|c|}
\hline Condition & Group & Rat ID & y0 & SE & $\mathrm{xc}$ & SE & $\mathbf{w}$ & SE & A & SE & WHM & Area & $\mathbf{R}^{2}$ \\
\hline \multirow[t]{11}{*}{ Baseline } & 6-OHDA & L8 & .054 & .022 & 31.377 & .472 & 12.538 & .605 & .969 & .036 & 29.524 & 30.462 & .977 \\
\hline & & MA5 & .154 & .023 & 34.040 & .531 & 11.408 & .670 & .873 & .039 & 26.864 & 24.974 & .968 \\
\hline & & G11 & .168 & .021 & 34.123 & .499 & 9.615 & .596 & .871 & .042 & 22.642 & 20.989 & .964 \\
\hline & & S16 & .179 & .033 & 32.05 & .798 & 11.654 & 1.003 & .853 & .056 & 27.444 & 24.918 & .931 \\
\hline & & $\mathrm{T} 17$ & .102 & .026 & 28.558 & .554 & 11.978 & .687 & .986 & .044 & 28.207 & 29.593 & .968 \\
\hline & & $\mathrm{T} 21$ & .055 & .017 & 30.598 & .367 & 10.52 & .444 & .965 & .032 & 24.773 & 25.447 & .983 \\
\hline & Sham & S4 & .088 & .018 & 32.101 & .372 & 13.146 & .488 & .958 & .027 & 30.956 & 31.581 & .987 \\
\hline & & $\mathrm{L} 12$ & .165 & .028 & 31.166 & .691 & 11.317 & .856 & .847 & .05 & 26.651 & 24.034 & .946 \\
\hline & & S13 & .047 & .025 & 38.073 & .54 & 9.237 & .639 & .958 & .052 & 21.752 & 22.193 & .956 \\
\hline & & S18 & .070 & .026 & 34.796 & .530 & 11.749 & .679 & .989 & .043 & 27.668 & 29.133 & .969 \\
\hline & & BFN19 & .121 & .024 & 34.352 & .532 & 11.733 & .680 & .890 & .039 & 27.628 & 26.189 & .969 \\
\hline \multirow[t]{11}{*}{ PL1 } & 6-OHDA & L8 & .068 & .01 & 29.392 & .525 & 12.556 & .664 & .409 & .017 & 29.566 & 12.871 & .973 \\
\hline & & MA5 & .209 & .018 & 35.968 & .946 & 10.839 & 1.181 & .375 & .031 & 25.525 & 10.201 & .897 \\
\hline & & G11 & .108 & .015 & 41.448 & 1.525 & 10.006 & 1.856 & .201 & .029 & 23.563 & 5.032 & .743 \\
\hline & & S16 & .223 & .026 & 29.887 & .840 & 10.448 & 1.012 & .658 & .050 & 24.604 & 17.226 & .914 \\
\hline & & T17 & .086 & .016 & 28.443 & .739 & 10.825 & .892 & .449 & .029 & 25.490 & 12.195 & .936 \\
\hline & & $\mathrm{T} 21$ & .059 & .009 & 30.546 & .273 & 10.463 & .330 & .714 & .018 & 24.637 & 18.725 & .990 \\
\hline & Sham & S4 & .109 & .006 & 28.102 & .207 & 9.519 & .243 & .651 & .013 & 22.418 & 15.54 & .994 \\
\hline & & L12 & .170 & .024 & 30.812 & .838 & 10.618 & 1.018 & .591 & .044 & 25.004 & 15.736 & .916 \\
\hline & & S13 & .109 & .033 & 37.116 & .692 & 11.019 & .872 & .963 & .058 & 25.948 & 26.596 & .944 \\
\hline & & S18 & .065 & .015 & 35.225 & .402 & 11.683 & .516 & .716 & .024 & 27.511 & 20.963 & .982 \\
\hline & & BFN19 & .155 & .025 & 33.969 & 1.144 & 11.295 & 1.439 & .447 & .043 & 26.597 & 12.658 & .861 \\
\hline \multirow[t]{11}{*}{ PL2 } & 6-OHDA & L8 & .028 & .008 & 31.798 & .210 & 10.652 & .257 & .738 & .014 & 25.084 & 19.700 & .994 \\
\hline & & MA5 & .081 & .019 & 35.391 & .604 & 13.088 & .816 & .597 & .028 & 30.819 & 19.59 & .964 \\
\hline & & G11 & .059 & .010 & 37.602 & .533 & 10.647 & .663 & .379 & .018 & 25.071 & 10.120 & .964 \\
\hline & & S16 & .060 & .017 & 30.782 & .447 & 13.697 & .590 & .785 & .026 & 32.255 & 26.965 & .982 \\
\hline & & T17 & .166 & .026 & 28.287 & .635 & 11.296 & .773 & .875 & .047 & 26.600 & 24.763 & .955 \\
\hline & & $\mathrm{T} 21$ & .041 & .007 & 31.699 & .207 & 9.622 & .246 & .749 & .015 & 22.657 & 18.068 & .994 \\
\hline & Sham & S4 & .102 & .031 & 33.042 & .765 & 11.524 & .964 & .812 & .052 & 27.137 & 23.466 & .936 \\
\hline & & L12 & .076 & .021 & 33.653 & .340 & 9.275 & .401 & 1.308 & .044 & 21.840 & 30.418 & .982 \\
\hline & & S13 & .095 & .025 & 35.631 & .515 & 8.893 & .602 & .992 & .053 & 20.942 & 22.114 & .958 \\
\hline & & S18 & .015 & .012 & 37.916 & .317 & 11.178 & .403 & .731 & .020 & 26.321 & 20.489 & .988 \\
\hline & & BFN19 & .190 & .037 & 31.623 & .764 & 10.338 & .925 & .996 & .069 & 24.344 & 25.810 & .927 \\
\hline
\end{tabular}

a general decrease in response output and a recovery in shams but not in rats infused with 6-OHDA. Also, when examining measures within groups, 6-OHDA showed significant decreases in relative response rates when compared to its corresponding baseline. Thus, we propose that motivation to respond and not temporal control was impaired by 6-OHDA lesions (Simpson et al., 2011).

The results of the present study are consistent with previous findings by Meck (2006) in the sense that peak times and peak rates are independent measures. Although the lesions employed by Meck were different than the ones conducted in the present study, they were frontal cortex lesions through aspiration (and their corresponding controls had their skulls removed), the results were somewhat similar in that temporal control measures were unchanged after the lesions when dopaminergic drugs were administered. In this experiment, Meck employed a PIP with a single interval (FI $40 \mathrm{~s}$ and peak trials of $130 \mathrm{~s}$ ) and did not present the effects of lesions alone on temporal control but showed results of three different dopaminergic drugs three months after the lesions were conducted.

In the study by Orduña et al. (2012) using a PIP with FI $30 \mathrm{~s}$ and 90 -s peak trials (separated by an ITI with mean of $45 \mathrm{~s}$ ), similarly there were no consistent changes in peak times as a function of duration of training (20 vs. 120 sessions) and of two doses (.5 vs. $1 \mathrm{mg} / \mathrm{kg}$ ) of d-amphetamine, but instead a widening of the response distribution. In the Orduña et al. study, a dopamine agonist increased measures of response rates, not timing. Such finding complements the observed changes in response rates (decreases), not of timing measures (e.g., peak time, PRP) obtained in the present study by lesions of DA neurons. 
Also similar to the present experiment, the study by Balci et al. (2010a) showed no significant changes in the peak times between a group of transgenic mice that underexpress the dopamine transporter (DAT) with high levels of extracellular dopamine and a group of littermate wildtypes both trained in a PIP with two target times (FI $30 \& 45 \mathrm{~s}$, with 120 -s peak trials). Olton et al. (1988) and Olton (1989) employed a PIP using two times (short, FI $10 \mathrm{~s}$ and long, FI $20 \mathrm{~s}$ ) and 130-s peak trials to examine effects of frontal cortex lesions in rats. In both studies Olton et al. and Olton state that there were no general temporal control impairments and only during the long intervals was there a rightward shift in the peak functions, which corresponds to the later responding than before the lesions. Olton acknowledges lesions of the PFC cannot be truly examined in isolation due to existing connections with other areas of the brain.

By increasing motivation (i.e., increasing reinforcement rate) in a PIP the response functions generated during the peak trials recovered to levels closer to those shown by control mice (Ward et al., 2009). Tentatively, lack of motivation in the presented study was demonstrated by lowered behavioral output and by degenerating the dopamine mesocortical pathway with 6-OHDA.

Thus far, we have pointed to procedural differences between similar studies and the present one: (1) the use of two intervals versus a single interval in the PIP, and (2) neuronal alterations conducted using different methods (i.e., aspiration, genetic manipulations, and 6-OHDA). Olton (1989) and Olton et al. (1988) found impairments in timing when using two instead of a single interval in a PIP, which was viewed as impairment of divided attention. When using a single interval in Olton's and the present experiment, no evident alterations in temporal control were observed. It could be said that undivided attention is not disrupted in this model of schizophrenia and that when sources of stimulation increase, as in adding another interval to a PIP, then control of such stimuli over behavior may become more challenging.

In terms of the different methods to generate neuronal alterations to model those present in schizophrenia, we contend that the 6-OHDA model may provide an advantage over using transgenic mice. The 6-OHDA model is much more specific in terms of the different anatomical sites where DA2 receptors are located. In the present experiment we limited the damage to DA neurons in the mesocortical pathway. With knock-out mice all dopaminergic neurons are affected, thus generally altering DA pathways including those more closely associated with motor systems such as the nigrostriatal pathway.

Just as no evident impairments of temporal control were found after 6-OHDA lesions in the MPFC in the present study, Martin-Iverson et al. (1986) found that the same types of lesions in the same location as in the present experiment did not disrupt intravenous self-administration of cocaine. Thus, these authors concluded that the MPFC may not play a critical role in the maintenance of cocaine self-administration. These authors also found an increase in DA turnover in the striatum and NAC which supports the compensatory response generated by this 6-OHDA model of schizophrenia (Li et al., 2010).

By reviewing the diversity of results obtained within this area of research and what is pertinent to the function of the mPFC, this structure is functionally heterogeneous, and it is difficult to study in isolation (Olton, 1989). The present study examined the role of the $\mathrm{mPFC}$ in the temporal organization of behavior. Although it has been shown by Valencia-Torres et al. $(2011,2012)$ that both PFC and NAC are involved in temporal discrimination, we conclude that the $\mathrm{mPFC}$ may structure future behavior and organize it, but it is known that temporal control involves the integration of multiple neural systems (Meck, 2006) not solely mPFC. We contend that in the present study it is possible that compensatory mechanisms from the NAC ( $\mathrm{Li}$ et al., 2010) may be sufficient to maintain temporal control undisrupted in a PIP with a single interval, thus the role of mesocortical dopamine may be more related to motivational aspects of behavior.

The fact that the difference in tyrosine hydroxylase counts between groups was not statistically significant represents a limitation of the present study; however, the focus of the present study was on examining behavioral measures and the effects of 6-OHDA on such measures as an animal model of schizophrenia. One reason for the lack of a significant difference could be that the dose of 6-OHDA infused was not high enough; other studies such as the study by Li et al. (2010) used a higher dose and obtained clearer differences after processing neural tissue. Another factor that may account for the lack of consistency between groups in this respect is the number of rats included in each group. We contend that despite this inconsistency, the present study has valuable contributions to the existing body of literature in that, to our knowledge, it is the first study to examine the effects of 6-OHDA lesions as a model of schizophrenia using a PIP. In addition, the present study included longer recovery periods both after lesions and postoperative training in a PIP, relative to most of the previous similar studies in this field (cf., Kostrzewa \& Jacobowitz, 1974). Another contribution of this study is that novel quantitative measures (i.e., AUC and PRPs) were used to examine changes in behavior as a function of neuronal alterations by 6-OHDA.

Because no impairments in temporal control were found in the present experiment, but instead what could be construed as diminished motivation to respond (or diminished behavioral output), these results may be potentially likened to some of the negative symptoms of schizophrenia (i.e., low motivation, avolition, cf. Li et al., 2010). Thus this 6-OHDA and PIP animal model may then be considered viable to examine negative and not solely cognitive symptoms of schizophrenia (Balci et al., 2010b; Simpson et al., 2011; Ward 2009). 


\section{References}

Balci, F., Ludvig, E. A., Abner, R., Zhuang, X., Poon, P., \& Brunner, D. (2010a). Motivational effects on interval timing in dopamine transporter (DAT) knockdown mice. Brain Research, 1325, 89-99.

Balci, F., Meck, W. H., Moore, H., \& Brunner, D. (2010b). Timing deficits in aging and neuropathology. In J. L. Bizon and A. G. Woods (Eds). Animal Models of Human Cognitive Aging (pp. 161201). New York: Humana Press.

Body, S., Cheung, T. H. C., Valencia-Torres, L., Olarte-Sànchez, C. M., Fone, K. C. F., Bradshaw, C. M., \& Szabadi, E. (2013). Pharmacological studies of performance on the free-operant psychophysical procedure. Behavioural Processes, 95, 71-89.

Carroll, C. A., O'Donnell, B. F., Shekhar, A., \& Hetrick, W. P. (2009). Timing dysfunctions in schizophrenia as measured by a repetitive finger tapping task. Brain Cognition, 71, 345-353.

Catania, C. (1991). Time as a variable in behavior analysis. In I. H. Iversen, \& K. A. Lattal (Eds.), Experimental analysis of behavior: Part I (pp. 1-19). New York: Elsevier Science.

Elvevåg, B., Weinberg, D. R., \& Goldberg, T. E. (2002). Short-term memory for serial order in schizophrenia: a detailed examination of error types. Neuropsychology, 15, 128-135.

Ferster, C. B., \& Skinner, B. F. (1957). Schedules of reinforcement. Copley: Acton, MA.

Heidbreder, C. A., \& Groenewegen, H. J. (2003). The medial prefrontal cortex in the rat: evidence for a dorso-ventral distinction based upon functional and anatomical characteristics. Neuroscience and Biobehavioral Reviews, 27, 555-579.

Jones, C. A., Watson, D. J. G., \& Fone, K. C. F. (2011). Animal models of schizophrenia. British Journal of Pharmacology, 164, 11621194

Kostrzewa, R. M. \& Jacobowitz, D. M. (1974). Pharmacological actions of 6-hydroxydopamine. Pharmacological Reviews, 26, 199-288.

Lejeune, H. (1990). Timing: Differences in continuity or generality beyond differences? In D. E. Blackman \& H. Lejeune (Eds.). Behaviour Analysis in Theory and Practice Contributions and Controversies (pp. 53-90). East Sussex, UK: Lawrence Erlbaum Associates.

Li, Chun-Rong, Huang, G., Sui, Z. Y., Han, E., and Chung, Y. (2010). Effects of 6-hydroxydopamine lesioning of the medial prefrontal cortex on social interactions in adolescent and adult rats. Brain Research, 1346, 183-186.

Martin-Iverson, M. T., Szostak, C., \& Fibiger, H. C. (1986). 6-hydroxydopamine lesions of the medial prefrontal cortex fail to influence intravenous self-administration of cocaine. Psychopharmacology, 88, 310-314.
Meck, W. H. (2006). Frontal cortex lesions eliminate the clock speed effect of dopaminergic drugs on interval timing. Brain Research, 1108, 157-197.

Myerson, J., Green, L., \& Warusawitharana, M. (2001). Area under the curve as a measure of discounting. Journal of the Experimental Analysis of Behavior, 76, 235-243.

Olton, D. S. (1989). Frontal cortex, timing and memory. Neuropsychologia, 27, 121-130.

Olton, D. S., Wenk, G. L., Church, R. M., \& Meck, W. H. (1988). Attention and the frontal cortex as examined by simultaneous temporal processing. Neuropsychologia, 26, 307-318.

Orduña, V., García, A., \& Bouzas, A. (2012). Evaluation of ratedependency and internal clock effects of d-amphetamine. Behavioural Processes, 90, 428-432.

Roberts, S. (1981). Isolation of an internal clock. Journal of Experimental Psychology: Animal Behavior Processes, 7, 242-268.

Saulsgiver, K. A., McClure, E. A., \& Wynne, C. D. L. (2006). Effects of d-amphetamine on the behavior of pigeons exposed to the peak procedure. Behavioural Processes, 71, 268-285.

Simpson, E. H. et al. (2011). Pharmacologic rescue of motivational deficit in an animal model of the negative symptoms of schizophrenia. Biological Psychiatry, 69, 928-935.

Sokolowski, J. D. \& Salamone, J. D. (1994). Effects of dopamine depletions in the medial prefrontal cortex on DRL performance and motor activity in the rat. Brain Research, 642, 20-28.

Tysk, L. (1983). Time estimation by healthy subjects and schizophrenic patients: a methodological study. Perceptual and Motor Skills, 56, 983-988.

Valencia-Torres, L., Olarte Sanchez, C. M., Body, S., Fone, K. C., Bradshaw, C. M., \& Szabadi, E. (2011). Fos expression in the prefrontal cortex and nucleus accumbens following exposure to retrospective timing tasks. Behavioral Neuroscience, 125, 202214.

Valencia-Torres, L., Olarte Sanchez, C. M., Body, S., Fone, K. C., Bradshaw, C. M., \& Szabadi, E. (2012). Fos expression in the orbital prefrontal cortex after exposure to the fixed interval peak procedure. Behavioural Brain Research, 229, 372-377.

Ward, R. D., Kellendonk, C., Simpson, E. H., Lipatova, O., Drew, M. R., Fairhurst, S., ... Balsam , P. D. (2009). Impaired timing precision produced by striatal D2 receptor overexpression is mediated by cognitive and motivational deficits. Behavioral Neuroscience, 123, 720-730.

Ward, R. D., Simpson, E. H., Kandel, E. R., \& Balsam, P. D. (2011). Modeling motivational deficits in mouse models of schizophrenia: Behavior analysis as a guide for neuroscience. Behavioural Processes, 87, 149-156. 